\title{
The Association between Time to Positivity and Staphylococcus Aureus Bacteremia in a Geriatric Population
}

Maillart $\mathrm{E}^{1 *}$, Karmali $\mathbf{R}^{2}$, Miendje Deyi VY³, Mascart $\mathbf{G}^{3}$ and Cherifi $\mathbf{S}^{1}$

${ }^{1}$ Infectious Diseases Unit, Department of Internal Medicine, Brugmann University Hospital, Brussels, Belgium

${ }^{2}$ Department of Internal Medicine, Brugmann University Hospital, Brussels, Belgium

${ }^{3}$ Department of Microbiology, Brugmann University Hospital, Brussels, Belgium

\begin{abstract}
Background: Time to Positivity (TTP) of blood cultures is defined as the time elapsed between the start of incubation and the automated alert signal indicating growth in the culture bottle.

This study evaluates the TTP of blood cultures in patients with Staphylococcus aureus Bacteremia (SAB) and assesses the relationship between TTP and mortality,

Methods: We performed a retrospective study involving adults who had SAB between May 2007 and May 2010 in a tertiary hospital. TTP was defined as the time between onset of incubation and growth detection using an automated blood culture system.

Results: A total of 167 SAB were evaluated. Patient's median age was 72 years (range, 18-95 years). The median TTP was $13.6 \mathrm{~h}$ (range, 3.6-95.2 h). Attributable mortality rate $(27.5 \%)$ was not related to the TTP $(P=0.558)$ nor to the comorbidities. Age $>60$ years was the only independent predictor of attributable mortality $(P<0.001)$. Univariate analysis revealed a significantly shorter TTP in persistent bacteremia, endovascular source of infection, catheter-related infection and in community-acquired SAB. A TTP $>11.3 \mathrm{~h}$ had a negative predictive value of $96.4 \%$ for endocarditis. TTP was not related to methicillin susceptibility nor resistance of Staphylococcus aureus $(P=0.934)$

Conclusion: In our elderly population, shorter TTP was significantly associated with a central source of infection and a persistent SAB. In addition for the first time, community-acquired SAB was associated with a shorter TTP. TTP may contribute to a better management of $S A B$ by facilitating clinical decisions, especially in endocarditis.
\end{abstract}

Keywords: Staphylococcus aureus; Bacteremia; Time to positivity; Endocarditis; Community-acquired

\section{Introduction}

Time to Positivity (TTP) of blood cultures is defined as the time elapsed between the start of incubation and the automated alert signal indicating growth in the culture bottle. TTP is related to the number of micro-organisms initially present in blood and their metabolism [1]. The higher the initial bacterial inoculum, the quicker the cut-off value for detection of positivity is reached $[1,2]$. TTP is an indirect marker of the bacterial load and is associated with a higher morbidity and mortality [3]. Differential TTP is used for the diagnosis of catheterrelated bacteremia $[2,4,5-7]$ and TTP is used for predicting the presence of Staphylococcus spp. bacteria in blood culture $[8,9]$.

Staphylococcus aureus is a major cause of bloodstream infection and is associated with significant morbidity and mortality [10]. Only a few studies have described the relationship between TTP and clinical outcome in S. aureus Bacteremia (SAB) [3,11-13].

Our aim was to describe the TTP in adult patients with SAB and to assess its relationship with different variables such as mortality, severity of $\mathrm{SAB}$, source of infection and methicillin resistance in order to improve the management of SAB based on TTP.

\section{Materials and Methods}

\section{Study population}

This retrospective study included all adult patients admitted to Brugmann University Hospital between $15^{\text {th }}$ May 2007 and $15^{\text {th }}$ May 2010 who presented at least one episode of SAB. The following data where collected: demographic characteristics; acquisition of SAB (nosocomial or community-acquired); screening of nasal carriage for methicillin-resistant S. aureus (MRSA); sources of bacteremia (primary, catheter-related, secondary); predisposing conditions and Charlson weighted index of comorbidity (Charlson WIC); microbiological data (antimicrobial resistance and TTP); criteria of severity (complications of bacteremia, control blood cultures, intensive car admission, department of hospitalization and length of stay); outcome (cure, relapse, death).

The Brugmann University Hospital is an 854-bed hospital in Brussels with around 27000 admissions per year. It has 32 intensive care unit beds, geriatric unit of 132 beds.

\section{Microbiological data}

A set of blood culture was performed by inoculating 7-10 mL of blood into BACTEC/F blood culture aerobic and anaerobic bottles. All the bottles were sent immediately at room temperature to the microbiology

*Corresponding author: Maillart E, Infectious Diseases Unit, Department of Internal Medicine, Brugmann University Hospital, 4 Place A. Van Gehuchten, 1020 Brussels, Belgium, Tel: 32-2 4772111; Fax: +32 2 4773075; E-mail: evelyne.maillart@chu-brugmann.be

Received July 30, 2012; Accepted September 27, 2012; Published September 29, 2012

Citation: Maillart E, Karmali R, Miendje Deyi VY, Mascart G, Cherifi S (2012) The Association between Time to Positivity and Staphylococcus Aureus Bacteremia in a Geriatric Population. J Med Microb Diagn 1:114. doi:10.4172/2161-0703.1000114

Copyright: ( 2012 Maillart E, et al. This is an open-access article distributed under the terms of the Creative Commons Attribution License, which permits unrestricted use, distribution, and reproduction in any medium, provided the original author and source are credited. 
laboratory and were loaded into the BACTEC 9240 system (Becton Dickinson, Cockeysville, Md). Incubation was performed until positive and for a maximum of seven days. Bottles with positive results were examined by Gram staining. In case of characteristics colonies of Grampositive cocci on direct examination, the standard coagulase reaction was performed with human plasma and their content was subcultured in a gelose. The gelose was incubated overnight at $37^{\circ} \mathrm{C}$ in aerobic atmosphere. If $S$. aureus was isolated, routine antibiotic susceptibility testing was performed including methicillin susceptibility determined by two methods: the disk diffusion method using an oxacillin disk and a cefoxitin one (Neo-Sensitabs, Rosco, Taastrup, Denmark) and a dilution technique on the automate Vitek-2 (bioMérieux, Marcy l'Etoile, France). In case of discordance between both techniques, the strain was sent to the Belgian reference laboratory for Staphylococcus spp. for molecular detection of the mecA gene.

\section{Definitions}

A set of blood culture was considered positive if at least one of both bottles was positive for $S$. aureus [14]. An episode of SAB was defined as $\geq$ one positive blood culture. The time of the SAB episode was the time of inoculation of the first positive blood culture [14]. Time to positivity of the blood culture was defined as the time between the start of incubation and the time that the automated alert signal indicating growth in the culture bottle sounded [11,12]. In case of multiple positive blood cultures inoculated within maximum two hours, the shortest TTP was taken into account $[1,3]$

$\mathrm{SAB}$ was considered as community-acquired if it occurred within $48 \mathrm{~h}$ after admission and as nosocomial thereafter [14].

Source of bacteremia was determined according to the definitions of the National Surveillance Program for Hospital Infections (NSIH). $\mathrm{SAB}$ was considered as primary, secondary or primary related to catheter [14]. We pooled the following sources into one group named as "central source": primary, primary related to catheter and secondary endovascular (endocarditis, arterio-venous fistula, vascular graft) [10]. Infective endocarditis was defined according to the modified Duke criteria [15]. Severity of underlying diseases present before the episode of SAB was assessed according to the Charlson WIC [16].

$\mathrm{SAB}$ was qualified as complicated if multiple clinical sources were identified simultaneously during the bacteremia or if there was metastatic infection [14]. Persistent SAB was defined if blood culture remained positive beyond $48 \mathrm{~h}$ of appropriate antibiotic treatment. A prolonged hospitalization was defined as a length of stay $\geq 15$ days, minimum time necessary to consider an uncomplicated $\mathrm{SAB}$ as optimally treated [17].

If an episode was pre-treated with an appropriate antibiotic (in-vitro activity against the isolated S. aureus strain) $72 \mathrm{~h}$ before inoculation, it was excluded from the analysis [18].

Cure was defined as a negative blood culture and/or absence of septic signs during seven days following the initiation of antibiotics [14]. SAB occurring more than 8 days after the end of one episode was considered as a new episode [14]. The attributable mortality was defined as death occurring with persistent bacteremia, with persistent signs of sepsis or occurring before the end of the episode of bacteremia without any other obvious cause [14].

\section{Statistical analysis}

Non-normally distributed quantitative variables were reported as median values with their range and their differences were evaluated with the Mann-Whitney test. Qualitative variables were assessed by Chi-square test or Fisher's exact test where applicable. Logistic regression was used for multivariate analysis. Receiver Operating Characteristics (ROC) curve was used to determine the cut-off values of TTP when univariate analysis (Mann-Whitney) was significant. The positive predictive value and negative predictive value were calculated according to the prevalence of that parameter in our cohort. A $\mathrm{P}<0.05$ was considered to be statistically significant.

\section{Results}

A total of 205 episodes of SAB were identified. Among these, 18 were excluded from the study because of incomplete or missing data and 20 episodes were excluded because they had received previous antibiotic treatment. A total of 167 episodes in 154 patients were reviewed for TTP analysis. Among ten patients with multiple episodes, one patient had four episodes, one presented three episodes and eight had two episodes.

\section{Characteristics of the study population}

The median age was 72 years (18-95 years) and 100 episodes (59.9\%) occurred in men. Among the 167 episodes of SAB, 112 (67.1\%)

\begin{tabular}{|c|c|c|c|c|c|}
\hline & $\mathbf{N}$ & $(\%)$ & & $\mathbf{N}$ & (\%) \\
\hline Patient groups & & & Cardiac echography & 98 & $(58.7)$ \\
\hline Geriatric $\geq 75$ years & 76 & $(45.5)$ & & & \\
\hline Non Geriatric & 91 & $(54.5)$ & Endocarditis & 21 & (12.6) \\
\hline Emergency & 22 & (13.2) & Native & 18 & $(10.8)$ \\
\hline Intensive care unit & 22 & $(13.2)$ & Biological & 2 & $(1.2)$ \\
\hline Internal medicine & 21 & $(12.8)$ & Mechanical & 1 & $(0.6)$ \\
\hline Surgery & 14 & (8.4) & & & \\
\hline Dialysis & 12 & $(7.2)$ & Primary Source & 22 & $(13.2)$ \\
\hline Charlson score & & & $\begin{array}{l}\text { Catheter-related } \\
\text { Source }\end{array}$ & 36 & $(21.6)$ \\
\hline $0-5$ & 56 & (33.5) & $\begin{array}{l}\text { Short term central } \\
\text { venous catheter }\end{array}$ & 18 & $(10.8)$ \\
\hline$>5-10$ & 83 & $(49.7)$ & $\begin{array}{l}\text { Long term central } \\
\text { venous catheter }\end{array}$ & 16 & $(9.6)$ \\
\hline$>10-15$ & 27 & $(16.2)$ & Others & 2 & $(1.2)$ \\
\hline \multirow[t]{2}{*}{$>15$} & 1 & $(0.6)$ & & & \\
\hline & & & Secondary Source & 109 & (65.3) \\
\hline Comorbidities & & & Respiratory & 26 & $(15.6)$ \\
\hline Cardiac insufficiency & 92 & (55.1) & Endovascular & 25 & $(15.0)$ \\
\hline Diabetes & 50 & (29.9) & Endocarditis & 18 & $(10.8)$ \\
\hline COPB & 50 & (29.9) & Cutaneous & 24 & $(14.4)$ \\
\hline Cirrosis & 6 & (3.6) & Osteoarticular & 13 & $(7.8)$ \\
\hline Dialysis & 26 & $(15.6)$ & Urinary & 12 & $(7.2)$ \\
\hline Neoplasia & 40 & $(24.0)$ & Others & 9 & $(5.4)$ \\
\hline Immunodeficiency & 30 & $(18.0)$ & & & \\
\hline Prothesis & 43 & $(25.7)$ & Outcome & & \\
\hline Obesity & 20 & $(12.0)$ & Cure & 91 & $(54.5)$ \\
\hline Long term catheter & 20 & $(12.0)$ & $\begin{array}{l}\text { Attributable } \\
\text { mortality }\end{array}$ & 46 & $(27.5)$ \\
\hline Renal insufficiency & 26 & $(15.6)$ & $\begin{array}{l}\text { Non-attributable } \\
\text { mortality }\end{array}$ & 13 & $(7.8)$ \\
\hline Ethylism & 26 & (15.6) & Relapse & 13 & $(7.8)$ \\
\hline Drug user & 8 & $(4.8)$ & Unknown & 4 & $(2.4)$ \\
\hline Peripheral arterial disease & 18 & $(10.8)$ & & & \\
\hline Stroke & 67 & $(40.1)$ & & & \\
\hline Others & 94 & (56.3) & & & \\
\hline
\end{tabular}

Table 1: Characteristics among 167 episodes of S. aureus bacteremia. 


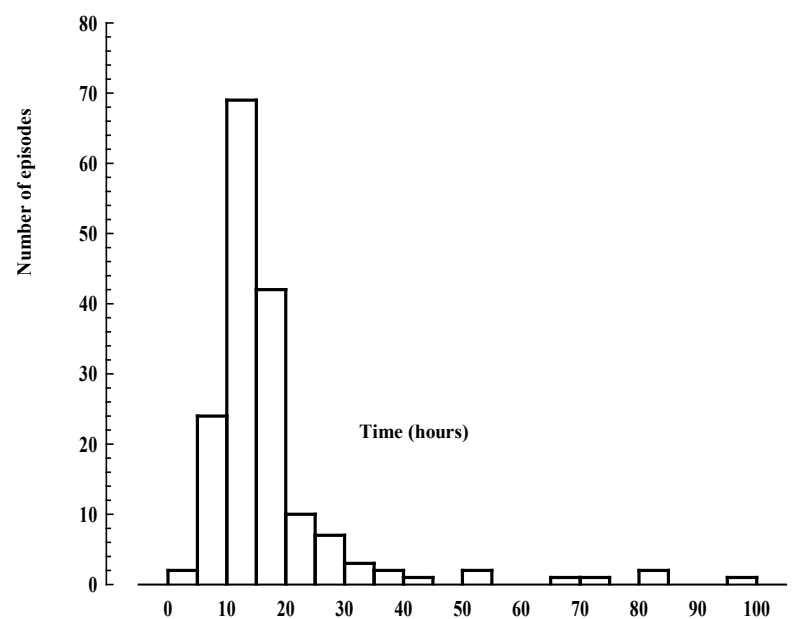

Figure 1: Distribution of TTP among 167 episodes of S. aureus bacteremia.

were caused by methicillin-sensible S. aureus (MSSA) and 72 (43.1\%) were classified as community-acquired. Screening of nasal carriage for MRSA was performed in $148(88.6 \%)$ episodes and in $46(31.1 \%)$ it was positive. Control blood cultures were performed in 34 (17.9\%) of the episodes $24 \mathrm{~h}$ after the beginning of an adequate antibiotic and in another $32(10.2 \%)$ after $48 \mathrm{~h}$ of adequate treatment. The overall median length of hospitalization was 30 days (0-237 days). The attributable mortality rate was $27.5 \%$ with a median age of 82 years at the time of death (31-94 years). Characteristics of the 154 patients with SAB are listed in table 1 .

\section{Time to positivity: Univariate analysis and predictive values}

The median TTP was $13.6 \mathrm{~h}$ (3.6 to $95.2 \mathrm{~h}$ ). Distribution of TTP is represented in figure 1 .

There was no significant relationship between TTP and attributable mortality $(P=0.558)$. Age $>60$ years did not influence the TTP $(P=0.256)$ but influenced the attributable mortality independently (OR: $8.93 ; 95 \%$ CI: 2.62-30.3; $\mathrm{P}<0.001)$. The logistic regression represented in table 2 showed that age was the only independent factor that influenced the attributable mortality $(\mathrm{P}<0.001)$. Attributable mortality was not related to any of the comorbidities studied.

Univariate analysis represented in table 3 revealed an association between a TTP $\leq 13.9 \mathrm{~h}$ and community-acquired $\mathrm{SAB}(P=0.032)$, a TTP $\leq 12.4 \mathrm{~h}$ and persistent bacteremia $(P=0.010)$, a TTP $\leq 12.7 \mathrm{~h}$ and an endovascular source of infection $(P=0.037)$ and a TTP $\leq 13.3 \mathrm{~h}$ and an intravascular catheter-related infection $(P=0.019)$. TTP $>11.3 \mathrm{~h}$ had a negative predictive value of $96.4 \%$ for endocarditis. The cut-off values of TTP are listed in table 4. Influence of different comorbidities on TTP was evaluated. Chronic Obstructive Pulmonary Disease (COPD), drug user and stroke significantly increased the TTP.

There was no relation between TTP and methicillin resistance: the median TTP was $13.55 \mathrm{~h}$ in MRSA bacteremias compared to $13.45 \mathrm{~h}$ in MSSA bacteremias $(P=0.934)$.

\section{Discussion}

In 1998, Blot et al. described that TTP of blood culture was related to the number of micro-organisms initially present in blood and their metabolism. They concluded that the higher the initial bacterial inoculum, the quicker the cut-off value for detection of positivity was reached [1]. Hence differential TTP was used in the diagnosis of catheter-related bacteremia $[2,7]$.

Further, studies showed that a TTP $\leq 14 \mathrm{~h}$ was correlated to attributable mortality in bacteremia varying from $11.9 \%$ to $18 \%$ $[3,11,12]$. In our study, attributable mortality $(27.5 \%)$ was higher than those reported in the literature but there was no significant association between TTP and attributable mortality. Two hypotheses could explain these results. First, our elderly population presented an important rate of comorbidities (Charlson score $\geq 5$ in $74 \%$ patients) that could have influenced mortality in SAB. However, we did not find a relation between attributable mortality and comorbidities as described in the literature $[11,13,16]$. The second hypothesis is the higher median age (72 years) in our cohort and the even higher age (82 years) among the deceased patients. In other studies, the population considered was heterogeneous including pediatric patients with a median age inferior to 62 years $[3,11,12]$. In fact, the only independent factor in our population that influenced the attributable mortality was age. Age has been described as correlated to attributable mortality in elderly patients with SAB $[19,20]$. It is possible that the mortality rate in our cohort was not only attributable to $\mathrm{SAB}$ but rather to age which might have annihilated the influence of TTP on mortality.

To our knowledge, our study is the first one to describe that a community-acquired bacteremia influences TTP with a cut-off TTP $\leq 13.9 \mathrm{~h}$. It has been reported that community-acquired SAB is more severe and complicated because the diagnosis is delayed due to the absence of major symptoms [21-23]. We hypothesize that communitydwelling patient, presenting to the emergency unit after a delay of three to four days of fever, when the bacteremia is already established with a large inoculum explaining a shorter TTP. In contrast, nosocomial bacteremia is often secondary or related to catheter infection and therefore more easily recognized [21]. Hospitalized patients are managed more promptly and this implies a smaller inoculum and hence a longer TTP.

Some comorbidities increased TTP (COPD, drug user and stroke). The reasons are not clear and warrant additional studies addressing specifically that question.

We observed also a relationship between TTP and one criteria of severity. In our study, $9 \%$ of the patients had persistent bacteremia, which was correlated to a TTP $\leq 12.4 \mathrm{~h}$. These results are similar to those reported by Khatib et al. [3], but their rate of persistent bacteremia was higher (32.1\%), probably because they used a different definition of persistent bacteremia.

As reported in the literature, we also found a relation between TTP and different sources of bacteremia such as endocarditis, endovascular, central and catheter-related infection $[9,12]$. Our cut-off values of TTP were in majority similar to those previously reported $[3,11]$. In our hospital, the knowledge of the negative predictive value in endocarditis and catheter-related bacteremia has a clinical impact on the medical approach of these situations.

Finally, TTP tended to be shorter, but not significantly in MSSA bacteremia when compared to MRSA bacteremia. Martinez et al. [9] described that median TTP in a MSSA bacteremia was significantly shorter than in MRSA bacteremia. In contrast, Ruimy et al. [8] reported a median TTP in MSSA longer than in MRSA bacteremia but it was not significant and without excluding pre-treated bacteremia. With these conflicting reports, one would think that TTP in MSSA and MRSA bacteremia is influenced by other factors than micro-organisms themselves such as the origin of bacteremia and prior antibiotic therapy 


\begin{tabular}{|c|c|c|c|c|c|c|c|c|c|c|}
\hline \multirow[t]{3}{*}{ Variable } & \multicolumn{5}{|c|}{ Unadjusted } & \multicolumn{5}{|c|}{ Adjusted } \\
\hline & \multicolumn{2}{|c|}{ Total } & \multirow[t]{2}{*}{ OR } & \multirow[t]{2}{*}{$(95 \% \mathrm{Cl})$} & \multirow[t]{2}{*}{ p } & \multicolumn{2}{|c|}{ Total } & \multirow[t]{2}{*}{ OR } & \multirow[t]{2}{*}{$(95 \% \mathrm{Cl})$} & \multirow[t]{2}{*}{$\mathbf{P}$} \\
\hline & $\mathbf{N}$ & (\%) & & & & $\mathbf{N}$ & $(\%)$ & & & \\
\hline Age $>60$ years & 48 & $(29.4)$ & 8.93 & $(2.62-30.3)$ & $<0.001$ & 115 & (70.6) & 9.09 & $(2.53-33.3)$ & $<0.001$ \\
\hline Male & 66 & $(40.5)$ & 1.34 & $(0.67-2.68)$ & 0.400 & 97 & $(59.5)$ & 0.91 & $(0.43-1.95)$ & 0.810 \\
\hline Charlson score $\geq 5$ & 44 & $(27.0)$ & 1.48 & $(0.66-3.30)$ & 0.343 & 119 & $(73.0)$ & 0.94 & $(0.38-2.35)$ & 0.893 \\
\hline MRSA & 109 & (66.9) & 1.45 & $(0.71-3.78)$ & 0.307 & 54 & (33.1) & 1.095 & $(0.52-2.32)$ & 0.813 \\
\hline Community-acquired & 94 & $(57.7)$ & 1.06 & $(0.53-2.12)$ & 0.868 & 69 & $(42.3)$ & 0.84 & $(0.397-1.78)$ & 0.649 \\
\hline
\end{tabular}

Table 2: Characteristics among 167 episodes of $S$. aureus bacteremia.

\begin{tabular}{|c|c|c|c|c|c|c|c|c|c|}
\hline \multirow[t]{3}{*}{ Variable } & \multicolumn{4}{|c|}{ Yes } & \multicolumn{4}{|c|}{ No } & \multirow[t]{3}{*}{$P$} \\
\hline & \multicolumn{2}{|c|}{ Total } & \multicolumn{2}{|c|}{ Median TTP } & \multicolumn{2}{|c|}{ Total } & \multicolumn{2}{|c|}{ Median TTP } & \\
\hline & $\mathbf{N}$ & (\%) & Hours & (range) & $\mathbf{N}$ & $(\%)$ & Hours & (range) & \\
\hline \multicolumn{10}{|l|}{ Characteristics } \\
\hline Age $>60$ years & 117 & $(71.3)$ & 13.3 & $(3.6-95.2)$ & 50 & (29.9) & 14.5 & $(7.3-65.1)$ & 0.256 \\
\hline Male & 100 & $(59.9)$ & 14.1 & $(3.6-95.2)$ & 67 & $(40.1)$ & 12.6 & $(4.4-69.9)$ & 0.074 \\
\hline Charlson score $\geq 5$ & 123 & $(73.7)$ & 13.6 & $(3.6-95.2)$ & 44 & (26.3) & 13.2 & $(7.9-52.6)$ & 0.740 \\
\hline MRSA & 55 & $(32.9)$ & 13.5 & $(7.9-81.5)$ & 112 & $(67.1)$ & 13.5 & $(3.6-95.2)$ & 0.934 \\
\hline Community-acquired & 72 & $(43.1)$ & 12.4 & $(7.2-69.9)$ & 95 & $(56.9)$ & 12.4 & $(7.2-69.9)$ & 0.032 \\
\hline Attributable mortality & 46 & $(27.5)$ & 13.2 & $(3.6-82.0)$ & 117 & $(70.1)$ & 13.6 & $(4.4-95.2)$ & 0.558 \\
\hline \multicolumn{10}{|l|}{ Comorbidities } \\
\hline Cardiac insufficiency & 92 & $(55.1)$ & 13.2 & $(3.6-74.8)$ & 75 & $(44.9)$ & 14.4 & $(7.2-95.2)$ & 0.053 \\
\hline Peripheral arterial disease & 18 & $(10.8)$ & 13.2 & $(4.4-28.0)$ & 149 & $(89.2)$ & 13.6 & $(3.6-95.2)$ & 0.541 \\
\hline Diabetes & 50 & $(29.9)$ & 12.4 & $(7.9-65.2)$ & 117 & $(70.1)$ & 13.8 & $(3.6-95.2)$ & 0.156 \\
\hline COPD & 50 & $(29.9)$ & 14.1 & $(3.6-95.2)$ & 117 & $(70.1)$ & 12.8 & $(4.4-81.5)$ & 0.040 \\
\hline Prothesis & 43 & $(25.7)$ & 15.4 & $(3.6-81.9)$ & 124 & $(74.3)$ & 13.2 & $(7.0-95.2)$ & 0.222 \\
\hline Stroke & 67 & $(40.1)$ & 15.4 & $(8.2-81.9)$ & 100 & $(59.9)$ & 12.9 & $(3.6-95.2)$ & 0.016 \\
\hline Drug user & 8 & $(4.9)$ & 21.7 & $(10.2-38.4)$ & 159 & $(95.2)$ & 13.3 & $(3.6-95.2)$ & 0.018 \\
\hline \multicolumn{10}{|l|}{ Criteria of severity } \\
\hline Complicated bacteremia & 32 & $(19.2)$ & 12.4 & $(7.2-38.4)$ & 135 & $(80.8)$ & 13.8 & $(3.6-95.2)$ & 0.088 \\
\hline Persistent bacteremia & 15 & $(9.0)$ & 11.6 & $(4.4-18.5)$ & 152 & $(91.0)$ & 13.8 & $(3.6-95.2)$ & 0.010 \\
\hline Intensive care unit stay & 51 & $(30.5)$ & 13.0 & $(3.6-52.6)$ & 116 & (69.5) & 13.6 & $(7.0-95.2)$ & 0.074 \\
\hline Prolonged hospitalization & 103 & $(61.7)$ & 14.0 & $(3.6-95.2)$ & 64 & $(38.3)$ & 12.7 & $(7.2-69.9)$ & 0.478 \\
\hline \multicolumn{10}{|l|}{ Source of bacteremia } \\
\hline Primary (a) & 22 & $(13.2)$ & 13.0 & $(4.4-23.2)$ & 145 & $(86.8)$ & 13.7 & $(4.4-23.2)$ & 0.435 \\
\hline Primary related to catheter (b) & 36 & $(21.6)$ & 11.6 & $(3.6-95.2)$ & 131 & $(78.4)$ & 13.9 & $(4.4-81.9)$ & 0.019 \\
\hline Secondary endovascular (c) & 25 & $(15.0)$ & 12.4 & $(7.2-32.7)$ & 142 & $(85.0)$ & 13.8 & $(3.6-95.2)$ & 0.037 \\
\hline Endocarditis & 18 & $(10.8)$ & 11.0 & $(7.2-32.7)$ & 149 & $(89.2)$ & 13.8 & $(3.6-95.2)$ & 0.006 \\
\hline Central $(a+b+c)$ & 83 & $(49.7)$ & 12.2 & $(3.6-95.2)$ & 84 & $(50.3)$ & 15.5 & $(7.0-81.9)$ & $<0.001$ \\
\hline
\end{tabular}

Table 3: Univariate analysis of different variables and TTP among 167 episodes of $S$. aureus bacteremia.

\begin{tabular}{|c|c|c|c|c|c|c|c|}
\hline \multirow{2}{*}{\begin{tabular}{|l|} 
Variable \\
Community-acquired bacteremia \\
\end{tabular}} & \multirow{2}{*}{$\begin{array}{c}\begin{array}{c}\text { TTP cut-off } \\
\text { (hours) }\end{array} \\
13.9\end{array}$} & \multicolumn{2}{|c|}{ AUC $(95 \% \mathrm{Cl})$} & \multirow{2}{*}{$\begin{array}{c}\begin{array}{c}\text { Sensibility } \\
(\%)\end{array} \\
66.7\end{array}$} & \multirow{2}{*}{$\begin{array}{c}\begin{array}{c}\text { Specificity } \\
(\%)\end{array} \\
56.8\end{array}$} & \multirow{2}{*}{$\begin{array}{r}\begin{array}{r}\text { PPV } \\
\text { (\%) }\end{array} \\
38.4\end{array}$} & \multirow{2}{*}{$\begin{array}{r}\text { NPV } \\
(\%) \\
80.9\end{array}$} \\
\hline & & 0.60 & $( \pm 0.04)$ & & & & \\
\hline Persistent bacteremia & 12.4 & 0.70 & $( \pm 0.07)$ & 66.7 & 61.8 & 10.0 & 96.7 \\
\hline Primary source related to catheter & 13.3 & 0.63 & $( \pm 0.05)$ & 66.7 & 58.3 & 21.1 & 91.3 \\
\hline Secondary endovascular source & 12.7 & 0.63 & $( \pm 0.06)$ & 60.0 & 57.7 & 12.1 & 93.5 \\
\hline Endocarditis & 11.3 & 0.70 & $( \pm 0.06)$ & 61.1 & 73.8 & 14.2 & 96.4 \\
\hline Central source & 15.4 & 0.68 & $( \pm 0.04)$ & 53.6 & 73.5 & 47.6 & 77.9 \\
\hline
\end{tabular}

Table 4: Sensibility, Specificity and Predictives Values of TTP.

[23]. On the other side, the genetic diversity of MRSA between countries with dominant MRSA spa types forming distinctive geographical clusters could explain the fact that in our study, methicillin resistance was not associated with a short TTP. MRSA strains in Belgium may be less virulent as compared to other clusters in the literature [24,25].

Our study had several limitations. Firstly it was limited by the retrospective nature of our analysis, the small size of our cohort and the lack of complete clinical data during the SAB except admission into intensive care unit; secondly, the volume of blood drawn in the bottles remained uncontrolled and may have influenced the TTP. Finally, all patients had not systematic blood culture control after introduction of adequate antibiotic therapy and therefore the number of persistent bacteremia might have been under-estimated.

In conclusion, this study suggests that attention should be paid to a short TTP because it is associated with a persistent bacteremia, an endovascular source of infection, a catheter-related infection and very interestingly with a community-acquired SAB. Another important information concerns the negative predictive value of $96.4 \%$ for a TTP 
Citation: Maillart E, Karmali R, Miendje Deyi VY, Mascart G, Cherifi S (2012) The Association between Time to Positivity and Staphylococcus Aureus Bacteremia in a Geriatric Population. J Med Microb Diagn 1:114. doi:10.4172/2161-0703.1000114

$>11.3 \mathrm{~h}$ in endocarditis. In our population, the high age of the patients and their comorbidities influence the TTP and modify its classically reported relation with attributable mortality. TTP, already useful for catheter-related bloodstream infection diagnosis is a relatively simple and fairly reliable method that can optimize the management and treatment of $\mathrm{SAB}$.

\section{Conflicts of Interest Statement}

The authors disclose any financial and personal relationships with other people or organizations that could inappropriately influence their work.

\section{References}

1. Blot F, Schmidt E, Nitenberg G, Tancrède C, Leclercq B, et al. (1998) Earlier Positivity of Central-Venous- versus Peripheral-Blood Cultures is Highly Predictive of Catheter-Related Sepsis. J Clin Microbiol 36: 105-109.

2. Blot F, Nitenberg G, Chachaty E, Raynard B, Germann N, et al. (1999) Diagnosis of catheter-related bacteraemia: a prospective comparison of the time to positivity of hub-blood versus peripheral-blood cultures. Lancet 354 : 1071-1077.

3. Khatib R, Riederer K, Saeed S, Johnson LB, Fakih MG, et al. (2005) Time to Positivity in Staphylococcus aureus Bacteremia: Possible Correlation with the Source and Outcome of Infection. Clin Infect Dis 41: 594-598.

4. Catton JA, Dobbins BM, Kite P, Wood JM, Eastwood K, et al. (2005) In situ diagnosis of intravascular catheter-related bloodstream infection: A comparison of quantitative culture, differential time to positivity, and endoluminal brushing. Crit Care Med 33: 787-791.

5. Chen WT, Liu TM, Wu SH, Tan TD, Tseng HC, et al. (2009) Improving diagnosis of central venous catheter-related bloodstream infection by using differential time to positivity as a hospital-wide approach at a cancer hospital. J Infect 59 : 317-323

6. Raad I, Hanna HA, Alakech B, Chatzinikolaou I, Johnson MM, et al. (2004) Differential Time to Positivity: A Useful Method for Diagnosing Catheter-Related Bloodstream Infections. Ann Intern Med 140: 18-25.

7. Safdar N, Fine JP, Maki DG (2005) Meta-Analysis: Methods for diagnosing intravascular device-related bloodstream infection. Ann Intern Med 142: 451 466 .

8. Ruimy R, Armand-Lefevre L, Andremont A (2005) Short time to positivity in blood culture with clustered gram-positive cocci on direct smear examination is highly predictive of Staphylococcus aureus. Am J Infect Control 33: 304-306.

9. Martinez JA, Pozo L, Almela M, Marco F, Soriano A, et al. (2007) Microbial and clinical determinants of time-to-positivity in patients with bacteraemia. Clin Microbiol Infect 13: 709-716.

10. Laupland KB, Ross T, Gregson DB (2008) Staphylococcus aureus Bloodstream Infections: Risk Factors, Outcomes, and the Influence of Methicillin Resistance in Calgary, Canada, 2000-2006. J Infect Dis 198: 336-343.

11. Marra AR, Edmond MB, Forbes BA, Wenzel RP, Bearman GM (2006) Time to Blood Culture Positivity as a Predictor of Clinical Outcome of Staphylococcus aureus Bloodstream Infection. J Clin Microbiol 44: 1342-1346.

12. Kim J, Gregson DB, Ross T, Laupland KB (2010) Time to blood culture positivity in Staphylococcus aureus bacteremia: Association with 30 -day mortality. J Infect 61: 197-204.

13. Sowden D, Anstey C, Faddy M (2008) Blood culture time to positivity as a predictor of mortality in community acquired Methicillin-susceptible Staphylococcus aureus bacteremia. J Infect 56: 295-296.

14. National Programme for Monitoring of Infections in Hospitals in Belgium (NSIH) (2000) Scientific Institute of Public Health, Department of Epidemiology Annual Report

15. Li JS, Sexton DJ, Mick N, Nettles R, Fowler VG Jr, et al. (2000) Proposed modifications to the Duke Criteria for the Diagnosis of Infective Endocarditis. Clin Infect Dis 30: 633-638.

16. Lesens O, Methlin C, Hansmann Y, Remy V, Martinot M, et al. (2003) Role of Comorbidity in Mortality Related To Staphylococcus aureus Bacteremia: A
Prospective Study Using the Charlson Weighted Index of Comorbidity. Infect Control Hosp Epidemiol 24: 890-896.

17. Liu C, Bayer A, Cosgrove SE, Daum RS, Fridkin SK, et al. (2011) Clinica practice guidelines by the infectious diseases society of America for the treatment of methicillin-resistant Staphylococcus aureus infections in adults and children. Clin Infect Dis 52: e18-e55.

18. Passerini R, Riggio D, Radice D, Bava L, Cassatella C, et al. (2009) Interference of antibiotic therapy on blood cultures time-to-positivity: analysis of a 5-year experience in an oncological hospital. Eur J Clin Microbiol Infect Dis 28: 95-98.

19. McClelland RS, Fowler VG Jr, Sanders LL, Gottlieb G, Kong LK, et al. (1999) Staphylococcus aureus Bacteremia Among Elderly vs Younger Adult Patients: comparison of clinical features and mortality. Arch Intern Med 159: 1244-1247.

20. Malani PN, Rana MM, Banerjee M, Bradley SF (2008) Staphylococcus aureus Bloodstream Infections: The Association Between Age and Mortality and Functional Status. J Am Geriatr Soc 56: 1485-1489.

21. Finkelstein R, Sobel JD, Nagler A, Merzbach D (1984) Staphylococcus aureus bacteremia and endocarditis: comparison of nosocomial and communityacquired infection. J Med 15: 193-211.

22. Willcox PA, Rayner BL, Whitelaw DA (1998) Community-acquired Staphylococcus aureus bacteraemia in patients who do not abuse intravenous drugs. QJM 91: 41-47.

23. Price J, Baker G, Heath I, Walker-Bone K, Cubbon M, et al. (2010) Clinical and Microbiological Determinants of Outcome in Staphylococcus aureus bacteraemia. Inter J Microbiol 2010: 654858

24. Grundmann H, Aanensen DM, Van den Wijngaard CC, Spratt BG, Harmsen D, et al. (2010) Geographic Distribution of Staphylococcus aureus Causing Invasive Infections in Europe: A Molecular-Epidemiological Analysis. PLoS Med 7: e1000215.

25. Denis O, Deplano A, Nonhoff C, De Ryck R, de Mendonça R, et al. (2004) National Surveillance of Methicillin-Resistant Staphylococcus aureus in Belgian Hospitals Indicates Rapid Diversification of Epidemic Clones. Antimicrob. Agents Chemother 48: 3625-3629. 